\title{
Dynamics of the M31 Planetary Nebula System
}

\author{
Xiaohui Hui \\ Center for Space Research, Massachusetts Institute of Technology, USA
}

\begin{abstract}
We made a spatially complete and kinematically unbiased survey of $M 31$ for planetary nebulae (PNs). The survey covers a projected area of 20 by $40 \mathrm{kpc}$ centered on $M$ 31. Subsequently, we measured the radial velocities of over $800 \mathrm{PNs}$ using the Hydra multi-object spectrograph on the KPNO 4-m telescope. With this large dataset, a detailed dynamical study of the stellar disk, bulge and halo populations in M 31 is currently underway. We are focusing on the dynamical transition between the disk, bulge and halo, and a comparison between the kinematics of the PNs and globular clusters.
\end{abstract}

\section{Introduction}

PNs are important test particles for the study of the dynamics of spiral galaxies. Kinematic information of the old stellar populations in both the bulge and disk usually come from the integrated absorption spectra. The rapid fall off in the surface brightness limits such observation to the very central region of spirals. Little is known about the dynamics of the old stellar disk and the stellar halo.

However, PNs are readily found in the outer disks and halos of spiral galaxies. Nolthenius and Ford (1987) detected 37 PNs in M31 between 15 and $30 \mathrm{kpc}$. They statistically separated the PNs into a disk population and a halo population. While the disk PNs at $20 \mathrm{kpc}$ have a rotation velocity $20 \mathrm{~km} / \mathrm{s}$ slower than $\mathrm{HI}$, possibly due to the asymmetrical drift, their limited data suggested that the halo PNs are dynamically similar to the halo globular clusters.

We greatly extended Nolthenius \& Ford's early work by detecting and measuring 800 new PN velocities in a spatially complete and kinematically unbiased sample in M31. In this paper, we report the photometric and spectroscopic observations, and the results of a preliminary analysis of the PN kinematics.

\section{Observations}

\subsection{IDENTIFICATION}

The planetary nebula survey was carried out on both the KPNO 4-m and 0.9-m telescopes. Observations were made with a pair of [O III] $\lambda 5007$ on-band/off-band interference

473

1. Appenzeller (ed.), Highlights of Astronomy, Vol. 10, 473-475.

(C) 1995 IAU. Printed in the Netherlands. 
filters and a Tek 2048 CCD camera. The survey covers a projected area of $20 \mathrm{kpc} \times 40$ kpc centered on M31 (the long axis of the survey is parallel to the M31's major axis). By matching candidates in the two surveys, we were able to positively exclude cosmic ray contamination and secure the identifications. In total, we found approximately 700 new PNs in the disk and halo of M31 (Ford et al. 1994). In addition, another 500 PNs in the central bulge were cataloged in several earlier surveys of M 31 (cf. Ciardullo et al. 1989).

\subsection{SPECTROSCOPY}

During a 4 night run in November 1993, spectra of approximately 800 PNs in M 31 were obtained with the bench mounted Hydra multi-object spectrograph on the KPNO $4-m$ telescope. We used the instrument combination of grating KPC-007 (632 lines/mm, $1^{\text {st }}$ order), blue fiber cable and T2KB CCD camera. The spectral dispersion was $\sim 0.99 \AA$ per pixel, and the FWHM of emission lines was $3.5 \AA$. The spectra covered wavelengths from $\lambda 4765$ to $\lambda 6800$. A few of the brightest PN emission lines, such as [O III] $\lambda 5007$, [OIII] $\lambda 4959, \mathrm{H}_{\alpha}$ and $\mathrm{H}_{\beta}$ fall into this range.

The PN survey area extends to $20 \mathrm{kpc}$ along both sides of the major axis and 10 kpc along the minor axis, covering approximately 4.5 square degrees on the sky. Since the Hydra field is only $40^{\prime}$ in diameter, we need more than 20 fields to cover the whole galaxy. Typically, each field has 1 to 215 minute exposures depending on the average $\mathrm{PN}$ brightness. The $\mathrm{Cu}-\mathrm{Ar}$ spectra were taken several times each night for the wavelength calibration. In total, spectra of over 800 PNs were acquired.

\section{Results}

The PN spectra were reduced using the IRAF package. The CCD images were bias subtracted and trimmed. Then the spectra were extracted using flat field exposure as templates. Finally, the spectra were calibrated into linear wavelength scale using the $\mathrm{Cu}$ Ar comparison spectra. Velocities were measured by a Gaussian fit to the [O III] $\lambda 5007$ emission line. By comparing velocities of those PNs with repeated measurements, we conclud that for about $50 \%$ of PNs which have peak counts over 400 , the measurement accuracy is about $5 \mathrm{~km} \mathrm{~s}^{-1}$. For PNs with less counts, the one sigma scattering is about $11 \mathrm{~km} \mathrm{~s}^{-1}$.

In Figure 1, all observed PN velocities are plotted against the distance along the photometric major axis of the galaxy. The systemic velocity of the PNs appears to be close to $-300 \mathrm{~km} \mathrm{~s}^{-1}$. The figure also shows a central bulge component with a velocity dispersion of $\sim 150 \mathrm{~km} \mathrm{~s}^{-1}$.

A detailed dynamical analysis of the stellar disk, bulge and halo populations is underway. We hope that a better understanding of the dynamical transition between the disk, bulge and halo will cast light on the subject of the formation and evolution of disk galaxies. 